\title{
Photometric distances to NGC 628 and its four companions ${ }^{\star}$
}

\author{
M.E. Sharina ${ }^{1}$, I.D. Karachentsev ${ }^{1,2}$ and N.A. Tikhonov ${ }^{1}$ \\ 1 Special Astrophysical Observatory, Russian Academy of Sciences, Zelenchukskaja,Stavropolsky kraj, 357147, Russia \\ 2 Visiting astronomer at the Astronomical Observatory of Capodimonte, via Moiariello 16, I-80131, Napoli, Italy
}

Received February 28; accepted April 5, 1996

\begin{abstract}
Based on photometry of the brightest blue stars we derived distance moduli for NGC 628 and its dwarf irregular companions: DDO 13, UGC 1171, UGC 1104, and K 10. The mean modulus of the group is estimated to be $29.46 \mathrm{mag}$ with a formal (internal) error of 0.11 . The total mass to luminosity ratio of $40 M_{\odot} / L_{\odot}$ seems sufficient for NGC 628 to keep its companions in bound orbits.
\end{abstract}

Key words: galaxies: NGC 628; distances and redshifts; irregular

\section{Introduction}

A large, seen face-on spiral M $74=$ NGC 628 belongs to the most prominent galaxies in the northern sky (Atlas of Galaxies, Sandage \& Bedke 1988). Nevertheless, its distance is still known with a surprisingly low accuracy. Thus, Sandage \& Tammann (1974a) derived for NGC 628 the distance modulus $(m-M)_{0}=31.46 \mathrm{mag}$, using for such purpose the sizes of its HII-regions. Later Bottinelli et al. $(1984,1985)$ determined its modulus as $26.60 \pm 0.62$ and $26.00 \pm 0.17 \mathrm{mag}$ respectively from the Tully-Fisher diagram and luminosity index. Perhaps, this is the most impressive case of disagreement (by one order of magnitude!) between the adherents of the long and short extragalactic scales.

In the wide neighbourhood of M 74 there are some dwarf galaxies, which may be considered as companions because of their radial velocities. For this group Tully (1987) estimated the virial mass to luminosity ratio to be 266 in solar units. However, according to Vennik (1984), its virial ratio is only $2 M_{\odot} / L_{\odot}$. The presented numbers manifest that our knowledge about the basic properties of even nearby galaxies seems sometimes too unreliable. In the present paper we aim at a better determination of the distance to NGC 628 and to its companions, based on photometry of their brightest stars. The classic method of distance measurements via the luminosity of the brightest red and blue supergiants (Sandage \& Tammann 1974bd; de Vaucouleurs 1978) has been less popular during the last years (Rozanski \& Rowan- Robinson 1994). However, in many cases, for instance, for face-on spirals or dwarf

Send offprint requests to: I.D. Karachentsev

* Tables 1 to 9 are available at the CDS via anonymous ftp to cdsarc.u-strasbg.fr (130.79.128.5) irregular galaxies the brightest stars method still remains a very useful way of distance estimation.

\section{Observations and data reduction}

Images of the central part of NGC 628 and its dwarf companions: DDO 13, UGC 1171, UGC 1104, K 10, and UGCA 20 were obtained at the prime focus of the $6 \mathrm{~m}$ telescope with a $580 \times 520$ pixels CCD under seeings FWHM $=1.4-1.9$ arcsec. The CCD has a pixel size of $24 \times 18$ microns and covers a field of $120 \times 80$ arcsec in the sky. We performed observations of the galaxies and of the standard stars (Landolt 1992) in the $B, V, R$ bands which are close to the Kron-Cousins system with a typical exposure of about $600 \mathrm{~s}$. The usual sequence of reductions: bias subtraction, flat- fielding, cosmetics etc., and star aperture photometry were performed with the PC VISTA package (Treffers \& Richmond 1989), modified by Georgiev (1990, 1996).

\section{Photometry results}

Judging from the literature the observed galaxies, except M 74, have not been resolved into stars as yet. Below we present our photometry for their brightest stars and use it to estimate distance moduli to the galaxies.

\section{1. $N G C 628=M 74$}

In addition to the CCD frames we have at our disposal also $B$ and $V$ plates obtained by us with the $6 \mathrm{~m}$ telescope on 9.10 .1986 under a seeing of 1.5 arcsec. The field of view of the plates, 12 arcmin, covers the whole apparent structure of the galaxy. Figure 1 reproduces the $V$-image of 


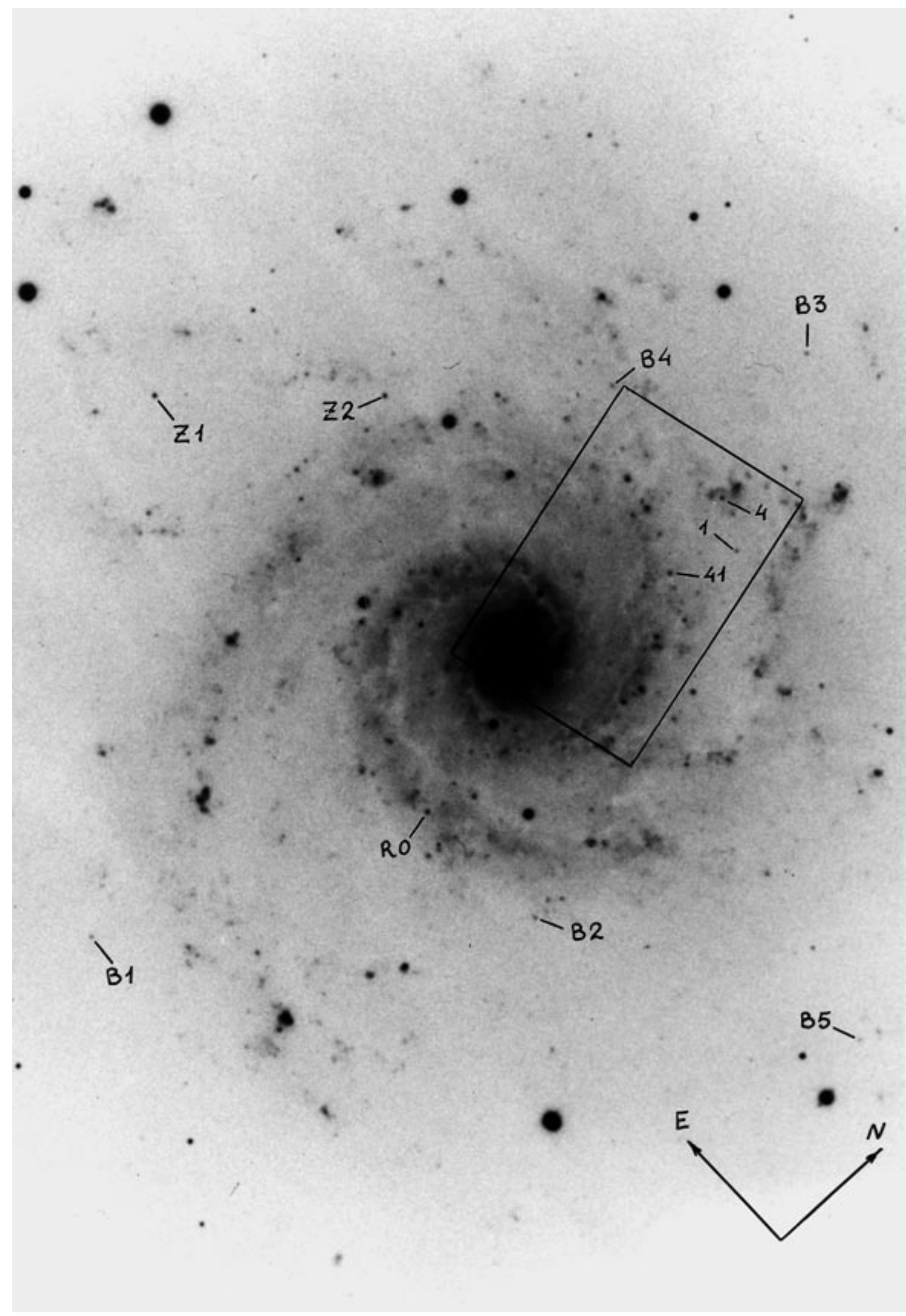

Fig. 1. NGC 628: $V$ plate obtained at the $6 \mathrm{~m}$ telescope. The numbers indicate the brightest blue stars and secondary photometric standards (Table 2). A rectangle indicates the region of $120 \times 80$ arcsec in size observed with the CCD 
NGC 628. The rectangle indicates the region viewed with the CCD. An essential part of it is occupied with a spiral arm, where blue supergiants are expected to be present. However, the inhomogeneous background and stellar crowding in the arm complicates aperture photometry here. For this reason the magnitude error exceeds 0.25 mag for stars with $B, V>22.5$ mag. The brightness pattern is especially intricate in the region close to the nucleus, which does not allow reliable photometry here. The results of measurements of $B, V$ magnitudes are presented in Table 1 for 98 stars. Its first column indicates the star number, the second and third columns give their rectangular coordinates in pixels, the fourth column contains $V$ - magnitudes, and the fifth shows the colour index. By a letter "D" in the last column we indicate the cases when an image appears somewhat diffuse or looks like a compact HII region on the H-alpha map by Belley \& Roy (1992).

The colour - magnitude (C-M) diagram for the measured objects is presented in Fig. 2. The majority of them are blue. Even the reddest star $\left(n^{\circ} 1\right)$ has a colour too blue to be considered as a red supergiant candidate, according to the criterion $B-V>1.6$ of Sandage \& Tammann (1974b-d). Among the brightest blue objects diffuse objects, marked by open circles in Fig. 2, dominate. The possibility of confusion between the true blue supergiants and compact blue star complexes seems to be the main reason for systematic errors, which influence the galaxy distance determination. According to our data, the three brightest blue stars in the studied region have an average apparent blue magnitude of 20.68 and an average color index $\langle B-V\rangle=-0.27$. Obviously,outside the region covered with the CCD frame one can find other, brighter candidates to the blue supergiants. To search for them, we used the $B, V$ plates obtained with the $6 \mathrm{~m}$ telescope. In addition we derived a sequence of secondary photometric standards in the galaxy uncrowded regions with more or less homogeneous background, using for that purpose the $1 \mathrm{~m}$ Zeiss telescope with a similar CCD chip. Two stars of the sequence are labeled in Fig.1 as Z1 and Z2. Finally, we selected the five brightest objects over the whole galaxy: B1,...B5; their apparent magnitudes are presented in Table 2. Following Sandage \& Tammann (1974b-d) and de Vaucouleurs (1978), we used the mean magnitude of the three brightest stars, $<B(3 \mathrm{~B})>=20.13$, as a distance indicator.

To derive the distance modulus we applied the relation

$$
<M_{\mathrm{b}}(3 \mathrm{~B})>=-0.51\left[<B(3 \mathrm{~B})>-B_{\mathrm{t}}\right]-4.14,
$$

which reflects the known correlation between the luminosity of the brightest blue stars and the magnitude of the parent galaxy, $B_{\mathrm{t}}$. The argument in the right side is independent of distance. The numerical parameters in (1) were calibrated (Piotto et al. 1992; Karachentsev \&
Tikhonov 1994) on galaxies whose distance moduli have been measured via Cepheids. According to PGC (Paturel et al. 1992), we adopt $B_{\mathrm{t}}=9.97 \mathrm{mag}$ for $\mathrm{M} 74$ and $A_{B}$ $=0.13$ for the galactic extinction, and derive a distance modulus $(m-M)_{0}=29.32 \mathrm{mag}$.

Fig. 2. Colour - magnitude diagram for the part of NGC 628 imaged with the CCD. Open circles refer to diffuse objects indicated in the last column of Table 1

Besides blue stars we undertook also a search for red stars throughout the whole galaxy body. Only one red enough star, R0, with $B-V=1.70$ and $V=19.52$ was found. However, it seems too bright $\left(M_{v}=-9.9\right)$ to be a red supergiant; it is evidently a foreground star. The blinking, used for selection of red stars on $B, V$ plates, allows us to distinguish stars above the limits $V<21.5$ and $B<23.0$. The apparent absence of red supergiants with the parameters $\left\langle M_{v}(3 \mathrm{R})\right\rangle=-7.8$ and $B-V>1.6$ leads to a distance modulus limit $(m-M)_{0}>29.1$, which is consistent with the previous estimate. With the derived modulus the total absolute magnitude of the galaxy, -19.5 mag, and also its standard linear diameter, $A_{25}=22 \mathrm{kpc}$, look quite typical of a Sc spiral.

\section{2. $U G C 1176=D D O 13=K 8$}

This irregular galaxy, located 1.2 degrees away from M 74, is included in the lists of DDO objects (van den Bergh 1966) and of low surface brightness systems (Karachentseva 1968). It has a rather large angular diameter, $a_{25}=4.6 \operatorname{arcmin}(\mathrm{PGC})$, and a radial velocity, $V_{\mathrm{h}}=$ $+629 \mathrm{~km} / \mathrm{s}$ (Briggs 1986), similar to the radial velocity of NGC $628(+656 \mathrm{~km} / \mathrm{s})$. It is first resolved into stars on our plate obtained with the $6 \mathrm{~m}$ telescope in 1985 . Figure 


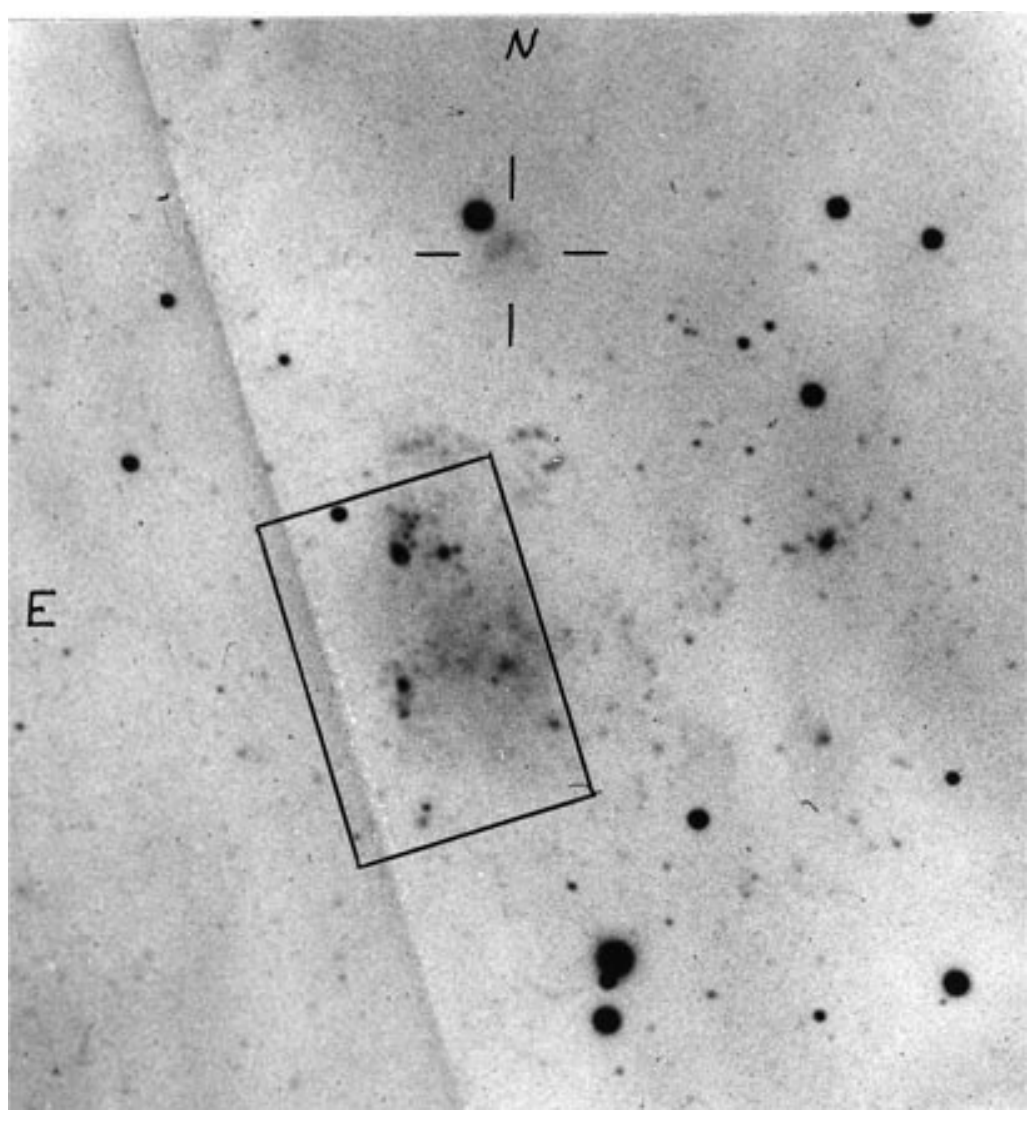

Fig. 3. DDO 13: blue plate. The rectangle shows the brightest $120 \times 80$ arcsec part of the galaxy observed with the CCD. The position of the HI- dwarf $0137+1541$ from Briggs (1986) is indicated by a cross. The band on the eastern side of the galaxy is an emulsion defect

3 shows its reproduction, where a rectangle of $120 \times 80$ arcsec in size indicates the CCD frame position covering the brightest part of the galaxy.

Our CCD observations of DDO 13 were obtained in the $B, V$ bands on December 5, 1993 under a seeing of $\mathrm{FWHM}=1.2$ arcsec. Figure 4 reproduces the $V$ image. Up to a limiting magnitude $B_{\text {lim }} \simeq 24$ mag more than 60 objects with colours in the range $[-0.4<B-V<1.6]$ are seen. About $1 / 3$ of them do not seem star-like. The three brightest blue stars are designated in Fig. 4 by B1, B2, B3. Their measured magnitudes are presented in Table 3. As secondary standards, we indicate here also other stars: A, B, C marked in Fig. 4. Therefore, we have $\langle B(3 \mathrm{~B})>$ $=21.94$ and $\langle B-V\rangle=+0.13$ for three candidate blue supergiants. Adopting for the galaxy $B_{\mathrm{t}}=14.37$ and $A_{\mathrm{b}}$ $=0.16(\mathrm{PGC})$, we derive a distance modulus $(m-M)_{0}$ $=29.78$. As brighter blue stars might be found outside our field, the present modulus should be considered as an upper limit.
Fig. 4. The brightest part of DDO 13: $V$ CCD frame. Secondary photometric standards and the three brightest blue stars are indicated by letters

\subsection{UGC 1171}

This curved boomerang-like dwarf with an angular diameter of 1.3 arcmin is located only 6 arcmin to the west of DDO 13. Their proximity was the cause of an erroneous HI velocity of $+667 \mathrm{~km} / \mathrm{s}$, given by Thuan \& Seitzer (1979). Carrying out HI observations with high angular resolution, Briggs (1982, 1986) obtained for UGC 1171 a heliocentric radial velocity of $+740 \mathrm{~km} / \mathrm{s}$.

We observed this galaxy with the CCD in the $B, V$ bands on December 30, 1994 under a seeing of 1.6 arcsec. Above $V=23.5$ there are about a dozen of blue and neutral-colour stars and knots. The three brightest blue stars are designated in Fig. 5 as B1, B2, and B3. The measured magnitudes for them as well for two nearby foreground stars are presented in Table 4 . Their mean apparent magnitude and colour are of $\langle B(3 \mathrm{~B})\rangle=22.10$ and $\langle B-V\rangle=+0.05$. We also determined the galaxy brightness in diaphragms of different size and after extrapolation found $B_{\mathrm{t}}=15.71$ and $(B-V)_{\mathrm{t}}=+0.42$. As a result, we 
deduce a distance modulus $(m-M)_{0}=29.34$ mag taking $A_{B}=0.16$.

\subsection{UGC 1104}

On the POSS prints the galaxy looks like a compact bluish object of regular shape. PGC only gives for it an optical radial velocity, $+669 \pm 50 \mathrm{~km} / \mathrm{s}$. According to Huchtmeier (1995), it is undetected in the HI line with the Effelsberg telescope in the range $[-600,+3900] \mathrm{km} / \mathrm{s}$. The CCD frames in the $B, V, R$ bands, obtained by us on December 30,1994, reveal a lot of stars and knots in its central part. The mean colour index of the galaxy increases towards the periphery. By these properties NGC 1104 resembles other nearby southern galaxies, NGC 1705 and NGC 2915 studied by Meurer et al., 1994. The $V$ frame of NGC 1104 is reproduced in Fig. 6. To show the structure of the central part in the presence of the strong gradient of brightness along the radius, we subtracted an image smoothed with a window of 25 pixels. The results of our photometry for the brightest blue stars and also for three foreground ones (A, B, C) are presented in Table 5. The brightest blue object, B0, is not quite star-like by appearance, and has been disregarded in the distance estimation. For the rest of the stars, B1-B3, we obtained their mean parameters: $\langle B(3 \mathrm{~B})\rangle=21.70,\langle B-V\rangle=$ +0.31 , and $\langle V-R>=+0.14$. A slightly yellowish colour of these stars is probably caused by systematic errors of photometry in the central crowded region of the galaxy against the strongly inhomogeneous background. According to our measurements the central part of the galaxy within a diameter of $20 \operatorname{arcsec}$ has $B-V=0.32$ and $V-R=$ 0.23 , but a wider region of 50 arcsec in diameter has 0.42 and 0.29 , respectively. With the integral apparent magnitude of the galaxy, $B_{\mathrm{t}}=14.48$, and $A_{B}=0.13$ its distance modulus from the three brightest blue stars is 29.39 mag. The observed absence of HI flux seems unusual for such a nearby blue compact galaxy.

\section{5. $K 10$}

This galaxy of low surface brightness from the list by Karachentseva (1968) has the standard angular diameter $a_{25}=1.3$ arcmin. Its radial velocity, $+791 \mathrm{~km} / \mathrm{s}$, was measured in the HI line by Briggs (1982) and confirmed by Huchtmeier (1995). The small HI line width, $W_{50}=$ $32 \mathrm{~km} / \mathrm{s}$, points out to a dwarf galaxy. We imaged K 10 with a CCD in the $B, V, R$ bands on December 29, 1994 with FWHM $=1.9$ arcsec. The $B$-frame is reproduced in Fig. 7. The three brightest blue stars in the galaxy body are designated as B1, B2 and B3. Table 6 contains their apparent magnitudes. Their mean magnitude, $<B(3 \mathrm{~B})>=$ 22.37 , and the total magnitude of the galaxy measured by us, $B_{\mathrm{t}}=16.28$, provide a distance modulus $(m-M)_{0}=$ 29.48. At such a distance the blue absolute magnitude of $\mathrm{K} 10$ is only -13.3 .

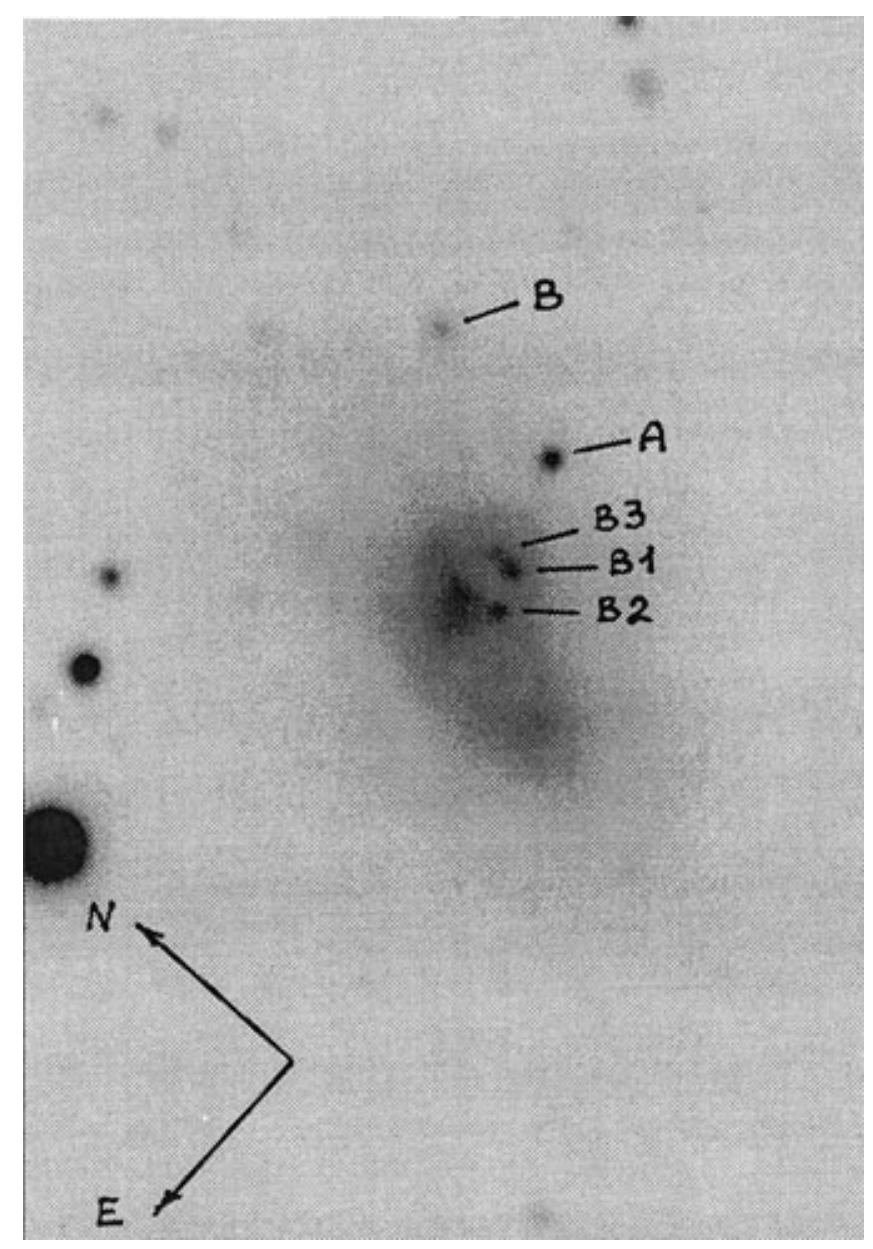

Fig. 5. CCD image of UGC 1171 in the $V$ band. Letters indicate the stars presented in Table 4

\subsection{UGCA 20}

At 4.5 degrees of M 74 there is another dwarf galaxy of low surface brightness, UGCA 20 , with a radial velocity +498 $\mathrm{km} / \mathrm{s}$. The galaxy is rather peculiar in shape, which differs significantly in the blue and red POSS prints. Probably, it consists of two separate elongated parts of $1.3 \times 0.3$ and $0.8 \times 0.2$ arcmin dimension, which are situated on opposite sides with respect to a remote pair of red galaxies. We obtained $V$ and $R$ images of the larger northern component of UGCA 20. A reproduction of the $R$ frame is presented in Fig. 8. Among about two dozen faint stars and knots seen in the object body we plot the three bluest stars in Fig. 8. Their magnitudes are listed in Table 7. Using a standard ratio of colour indices, $B-V=1.8(V-R)$, we obtain $\left\langle B(3 \mathrm{~B})>\simeq 22.7 \mathrm{mag}\right.$ for them. With $B_{\mathrm{t}}=15.9$ this gives us a distance estimate $(m-M)_{0} \simeq 30.2 \mathrm{mag}$ somewhat larger than for the other galaxies. Obviously, this rather unusual object needs more elaborate studying. 
Fig. 6. The $V$ image of UGC 1104 after subtraction of the background smoothed with a window of 25 pixels. North at the top, East on the left

\section{Group membership and orbital masses}

As it was mentioned above, differences in the virial mass estimates, given by different authors for the group around NGC 628, exceed two orders. Of course, this is mainly due to the problem of the membership in this group. Vennik (1984) considered the following objects: NGC 628, UGC 1104, UGC 1171 and UGC 1176 as the group members, which is confirmed by our measurements of their distances. Tully (1987) added other galaxies: NGC 660, UGC 1195, UGCA 20, DDO 13 and DDO 10 in the group M 74 (the group No. $17-4$ in his catalog (Tully 1988)), which led to increasing the group dimension, as well as its radial velocity dispersion.

To distinguish objects associated with NGC 628, we followed the approach proposed by Bahcall \& Tremaine (1981). By a group of galaxies is implied a system of companions which move around the main massive body along Keplerian orbits. According to the criterion of Karachentsev (1994), galaxies with masses $M_{k}$ and ve-
Fig. 7. The $B$ frame of the galaxy K 10. The three brightest blue stars are indicated

locities $V_{k}$ around a galaxy of mass $M_{1}$ and velocity $V_{1}$ are companions, if they satisfy the conditions

$$
\begin{gathered}
g_{1 k}=\left(V_{1}-V_{k}\right)^{2} \cdot R_{1 k} / 2 G \cdot\left(M_{1}+M_{k}\right)<1, \\
t_{1 k}=H \cdot R_{1 k}^{3 / 2} /\left[G\left(M_{1}+M_{k}\right)\right]^{1 / 2}<1 .
\end{gathered}
$$

Here $R_{1 k}$ is the projection of the linear separation between galaxies, $G$ is the gravitation constant, and $H$ is the Hubble parameter assumed here to be $75 \mathrm{~km} \mathrm{~s}^{-1} \mathrm{Mpc}^{-1}$. The upper relation indicates a negative total energy for each pair, and the lower one implies that its orbital period is less than the Hubble time, $H^{-1}$.

Some basic parameters of the galaxies in the vicinity of M 74 are collected in Table 8. Its lines give: (1) galaxy name, (2) morphological type, (3) standard angular diameter $a_{25}$ in arcmin, (4) apparent axis ratio, (5) total blue magnitude, (6) colour corrected for galactic extinction, (7) measured radial velocity, (8) HI line width measured at $50 \%$ level of the maximum, (9) radial velocity corrected 
Fig. 8. The $R$ frame of the brightest (northern) part of UGCA 20

for the Sun motion with respect to the Local Group centroid (Karachentsev \& Makarov 1996), and (10) measured distance. The quantities not taken from PGC (Paturel et al. 1992), but measured by ourselves, are noted by "c".

Applying criteria (2) and (3) to M 74 and to the galaxies close to it, we obtain the results summarized in Table 9. Here the total mass of each galaxy, $M$, was evaluated as

$$
M=\kappa \cdot(8 G)^{-1} \cdot A_{25} \cdot W_{50}^{2} /\left[1-(b / a)^{2}\right]
$$

$A_{25}$ being the standard linear diameter, and the amplitude of internal motions, corrected for the galaxy inclination. When the galaxy is viewed almost face-on $(b / a>0.8)$, we derive the mass from the luminosity $L$ and morphological type $T$

$$
M / L=\kappa \cdot(8-0.4 \cdot T) \cdot\left(M_{\odot} / L_{\odot}\right),
$$

These estimates are marked in Table 9 with an asterisk. For the dimensionless parameter $\kappa=M / M_{25}$ we adopt the value $\kappa=2.5$ in agreement with the observational data of Wevers (1984) and Begeman (1987). The luminosities and diameters of the galaxies were corrected for galactic extinction and inclination as adopted in the PGC.

The present data show that all dwarf objects in the considered region, except for UGCA 20, are situated inside the sphere of gravitational influence of two normal spiral galaxies: NGC 628 and NGC 660. They are galaxies of almost the same mass, and they have the same number of companions. Here the dwarf system K 10 is a companion of NGC 660, not NGC 628. The positions of the galaxies is shown in Fig. 9. Given to their dimension $(\sim 150 \mathrm{kpc})$ and velocity dispersion $\left(\sim 50 \mathrm{~km} \mathrm{~s}^{-1}\right)$ both quartets are not exceptional among nearby groups of galaxies (Karachentsev 1996). The median value of the dimensionless potential factor $g_{1 k}$ for the companions of NGC 628 and NGC 660 are 0.17 and 0.24 , respectively. According to Karachentsev (1994), the expected median value of $g_{1 k}$ is equal to 0.10 for a system of Keplerian satellites, which move in circular orbits orientated arbitrarily. The median decreases smoothly with increasing orbit eccentricity, $e$, and reaches a value of 0.063 with $e=0.6$. A comparison of the observed median with the expected one shows us that the virial ("orbital") mass of each group is about 3-4 times the mass of their main galaxy.

Fig. 9. The groups NGC 628 and NGC 660 in galactic coordinates. The companions which satisfy conditions (2),(3) are linked to the main galaxies with lines

\section{Discussion}

Our distance modulus estimates for four galaxies in the group NGC 628 have a mean value of 29.46 mag and a surprisingly low standard deviation $\sigma(m-M)=$ 
Table 8. Properties of the galaxies around NGC 628

\begin{tabular}{|lccccccc|ccc|}
\hline \multicolumn{2}{|c}{ Parameter } & U 1104 & NGC 628 & U 1171 & DDO 13 & UGCA 20 & K 10 & U 1195 & U 1200 & N 660 \\
\hline $\mathrm{T}$ & & 10 & 5 & 10 & 10 & 10 & $10 \mathrm{c}$ & 10 & 10 & 1 \\
$a_{25}$, & arcmin & 1.0 & 10.4 & 1.3 & 4.6 & $1.4 \mathrm{c}$ & $1.3 \mathrm{c}$ & 3.4 & 2.0 & 8.3 \\
$b / a$ & & 0.63 & 0.92 & 0.92 & 0.80 & $0.23 \mathrm{c}$ & $0.85 \mathrm{c}$ & 0.32 & 0.68 & 0.38 \\
$B_{\mathrm{t}}$ & & $14.48 \mathrm{c}$ & 9.97 & $15.71 \mathrm{c}$ & 14.37 & $15.9 \mathrm{c}$ & $16.28 \mathrm{c}$ & 13.44 & 13.81 & 11.7 \\
$(B-V)_{0}$ & & $0.38 \mathrm{c}$ & 0.52 & $0.38 \mathrm{c}$ & 0.51 & - & $0.42 \mathrm{c}$ & - & - & 0.74 \\
$V_{\mathrm{h}}$, & $\mathrm{km} \mathrm{s}^{-1}$ & +669 & +656 & $+740 \mathrm{c}$ & +629 & +498 & +790 & +774 & +808 & +853 \\
$W_{50}$, & $\mathrm{km} \mathrm{s}^{-1}$ & - & 53 & 23 & 38 & 61 & 32 & 127 & 128 & 306 \\
$V_{0}$, & $\mathrm{km} \mathrm{s}^{-1}$ & +850 & +825 & +907 & +796 & +676 & +954 & +933 & +963 & +1010 \\
$D$, & $\mathrm{Mpc}$ & 7.55 & 7.32 & 7.35 & 9.04 & $11 \mathrm{c}$ & 7.88 & - & - & - \\
\hline
\end{tabular}

Table 9. NGC 628 and NGC 660 with their companions

\begin{tabular}{|lccccc|cccc|}
\hline \multicolumn{2}{|c}{ Parameter } & NGC 628 & DDO 13 & U 1104 & U1171 & NGC 660 & U 1200 & U 1195 & K 10 \\
\hline$A_{25}$, & $\mathrm{kpc}$ & 22.6 & 12.0 & 2.1 & 2.8 & 18.3 & 5.0 & 7.2 & 2.9 \\
$(M / L)_{k}$, & $(M / L)_{\odot}$ & $15.0^{*}$ & 12.8 & $10.0^{*}$ & $10^{*}$ & 33.1 & 23.1 & 9.8 & $10^{*}$ \\
$M_{k}$, & $10^{10} \mathrm{M}_{\odot}$ & 14.3 & 0.35 & 0.19 & 0.07 & 14.50 & 1.10 & 0.94 & 0.03 \\
$V_{1}-V_{k}$, & $\mathrm{km} \mathrm{s}^{-1}$ & 0 & 30 & -25 & -82 & 0 & 46 & 77 & 56 \\
$R_{1 k}$, & $\mathrm{kpc}$ & 0 & 119 & 354 & 94 & 0 & 76 & 57 & 325 \\
$g_{1 k}$ & & 0 & 0.08 & 0.17 & 0.52 & 0 & 0.12 & 0.24 & 0.62 \\
$t_{1 k}$ & 0 & 0.13 & 0.64 & 0.09 & 0 & 0.06 & 0.04 & 0.48 \\
\hline
\end{tabular}

0.19 mag. This result together with a small scatter of moduli, $0.43 \mathrm{mag}$, for the members of the group M 101 (Karachentsev et al. 1994), and the components of the pair NGC 672 + IC 1727, $0.19 \mathrm{mag,} \mathrm{(Tikhonov} \mathrm{\&} \mathrm{Droz-}$ dovsky 1996) shows that the method of brightest stars is able to give distance estimates with an accuracy of 0.4 mag when applied to the late type galaxies. Therefore, if a group contains a sufficient number of irregular galaxies, the brightest stars method allows to measure the group distance with an almost "Cepheid precision". Adopting for the group M 74 a mean corrected radial velocity, $+844 \pm$ $24 \mathrm{~km} \mathrm{~s}^{-1}$, and a mean distance, $7.8 \pm 0.9 \mathrm{Mpc}$, we obtain a local value of the Hubble parameter, $H=108$ $\mathrm{km} \mathrm{s}{ }^{-1} \mathrm{Mpc}^{-1}$, with a formal error of $12 \%$. Note that the group is situated near the Supergalactic equator $\left(\mathrm{SGB}=-5^{\circ}\right)$ and is $17^{\circ}$ away from the Virgo core. Another group of 7 irregular galaxies around M 101, studied by us, has the mean values: $\left\langle V_{0}\right\rangle=+380 \pm 21$ $\mathrm{km} \mathrm{s}^{-1},\langle D>=6.6 \pm 1.0 \mathrm{Mpc}$, and $H=58 \mathrm{~km}$ $\mathrm{s}^{-1} \mathrm{Mpc}^{-1}$ (Karachentsev et al. 1994). The group M 101 is $46^{\circ}$ away the Virgo, and $23^{\circ}$ above the Supergalactic plane. Such a significant difference in the Hubble parameter estimates points out, apparently, to the presence of local deviations from the isotropic cosmic expansion. However, the standard spherically-symmetric model of Virgocentric flow (Kraan-Korteweg 1986) can explain only a small part $(\sim 25 \%)$ of the difference.

In Table 8 the data observe the large difference in brightness between NGC 628 and the rest of the members of the group. More than $97 \%$ of the total luminosity of the quartet is concentrated in its main component. As it was noted by Karachentsev (1996), the nearby groups with only one dominating galaxy do not normally contain elliptical and spheroidal members. This is the case for the M 74 and M 101 groups. A special search for spheroidal galaxies, undertaken on the POSS-II films by Karachentseva \& Karachentsev (1997), reveals no candidates in a wide vicinity of NGC 628 . Only one diffuse object of low surface brightness with an angular diameter of 0.3 arcmin and $B=19.3 \mathrm{mag}$ was found not far from DDO 13. Its coordinates correspond to the HI "Dw $0137+1541$ " discovered by Briggs (1986) with $V_{\mathrm{h}}=+750 \mathrm{~km} \mathrm{~s}^{-1}$. Its position indicated by the cross in Fig. 3 .

We noted above, that the dwarf objects in the considered region are concentrated around two normal spirals: NGC 628 and NGC 660. This association of two bright galaxies looks common in loose systems of galaxies. For instance, among the nearest 6 groups, considered by Karachentsev (1996), 4 groups show a tight association: the Local Group and M 31, NGC 5128 and NGC 5236.

It should be emphasized that considering such binary structures as a single gravitationally bound system leads to significant overestimation of its virial mass. For example,instead of the virial mass to luminosity ratio $335 M_{\odot} / L_{\odot}$, obtained by Tully (1987) for the whole $\{$ NGC $628+660\}$ aggregate with $D=7.8 \mathrm{Mpc}$, we derive individual ratios: $40 M_{\odot} / L_{\odot}$ and $126 M_{\odot} / L_{\odot}$ for the quartets NGC 628 and NGC 660 separately. The last estimates are based on statistics of Keplerian orbits assuming mean eccentricity $e=0.6$. Certainly, these quantities are 
poorly defined because of the poor statistics. However, removal or inclusion of a single member of the groups only changes the median of $g_{1 k}$ by a factor of $2-3$. In general the virial mass estimates for the groups, selected by conditions (2), (3), tend to be several times lower than those from the Tully (1987) catalog.

\section{Concluding remarks}

The bright spiral galaxy NGC 628 has been the subject of numerous studies in different aspects (von Hippel \& Bothun 1990; Natali et al. 1992; Kampuis \& Briggs 1992; Ivanov et al. 1992). Nevertheless, its distance was known with an unacceptably low accuracy: two distance modulus estimates published by Sandage \& Tammann (1974) and Bottinelli et al. $(1984,1985)$, differ from each other by more than 5 magnitudes.

Using large-scale images of NGC 628 obtained by us in the $B, V$ bands, we attempted a search and photometry of the brightest blue stars in the galaxy. Using the mean magnitude of the three brightest blue supergiants, we derived a distance modulus $(m-M)_{0}=29.32$. Using the same approach, we determined the distance moduli for the dwarf irregular companions of NGC 628: DDO 13, UGC 1171, UGC 1104 and K 10. The average modulus for them, $29.50 \pm 0.10 \mathrm{mag}$, agrees well with the modulus of the main galaxy in the group. As noted by McCall et al. (1985) and McCall (1995), the best agreement between the global properties of NGC 628 and its morphological type can be achieved with the modulus $29.3 \pm 0.3$. It also fits with the characteristic dimension of associations in NGC 628 (Ivanov et al. 1992).

A similar situation arises in the group M 101. For this face-on Sc galaxy the distance modulus from its brightest stars is $29.19 \mathrm{mag}$, and the average modulus for its seven irregular dwarf companions is $29.08 \pm 0.17$ (Karachentsev et al. 1994). A recent measurement of the modulus of M 101 via Cepheids (Alves \& Cook 1995) yields $(m-M)_{0}=29.08 \pm 0.13$, which is in excellent agreement with our data.

Although situated almost at the same distance $(7.8 \pm 0.9 \mathrm{Mpc}$ and $6.6 \pm 1.0 \mathrm{Mpc})$, the groups around $\mathrm{M} 74$ and M 101 have very different mean radial velocities: $+844 \pm 24 \mathrm{~km} / \mathrm{s}$ and $+380 \pm 21 \mathrm{~km} / \mathrm{s}$ with respect to the Local Group centroid. Such a difference seems too high to be explained in the framework of a spherically symmetric Virgocentric flow model (Kraan-Korteweg 1986). The observed difference in the Hubble parameter: 108 $\mathrm{km} \mathrm{s}^{-1} \mathrm{Mpc}^{-1}$ toward NGC 628 and $58 \mathrm{~km} \mathrm{~s}^{-1} \mathrm{Mpc}^{-1}$ toward M 101, may be caused by a slower anisotropic expansion of the Local cloud of galaxies in the directions of the poles (Karachentsev \& Makarov 1996).

We suggest that DDO 13, UGC 1171, and UGC 1104 are physical companions of NGC 628 , whereas the dwarf galaxy K 10 belongs to another nearby quartet with NGC 660 as its main member. Both groups, separated by a pro- jected distance of $0.5 \mathrm{Mpc}$, seem to be dynamically independent systems with a moderate virial mass excess. According to our estimate, their mass to luminosity ratio are $40 M_{\odot} / L_{\odot}$ and $126 M_{\odot} / L_{\odot}$, respectively, only $12-36 \%$ of the virial ratio obtained by Tully (1987) for the whole complex of galaxies.

Acknowledgements. The authors are indebted to Zwetan Georgiev for useful discussions. This work was supported by the ESO C\&EE Programme Grant A-02-016.

\section{References}

Alves D.R., Cook K.H., 1995, AJ 110, 192

Bachcall J., Tremaine S., 1981, ApJ 244, 805

Begeman K., 1987, Ph.D. Thesis, Groningen

Belley J., Roy J.R., 1992, ApJS 78, 61

Bottinelli L., Gouguenheim L., Paturel G., de Vaucouleurs G., 1984, A\&AS 56, 381

Bottinelli L., Gouguenheim L., Paturel G., de Vaucouleurs G., 1985, A\&AS 59, 43

Briggs F.G., 1982, ApJ 259, 544

Briggs F.G., 1986, ApJ 300, 613

de Vaucouleurs G., 1978, ApJ 224, 710

Georgiev Ts.B., 1990, Asrofiz. Issled (Izv.SAO) 30, 127

Georgiev Ts.B., 1996, Bull. Spets. Astrophys. Obs 39, (in press)

Huchtmeier W., 1995, (private communication)

Ivanov G.R., Popravko G., Efremov Y.N., Tikhonov N.A., Karachentsev I.D., 1992, A\&AS 96, 645

Kamphuis J., Briggs F., 1992, A\&A 253, 335

Karachentseva V.E., 1968, Publ. Byurakan Obs. 39, 61

Karachentseva V.E., Karachentsev I.D., 1997, (in preparation)

Karachentsev I.D., 1996, A\&A 305, 33

Karachentsev I.D., 1994, A\&Ap Trans. 6, 3

Karachentsev I.D., Kopylov A.I., Kopylova F.G., 1994, Bull. Spets. Astrophys. Obs. 38, 15

Karachentsev I.D., Makarov D.I., 1996, AJ 111, 794

Karachentsev I.D., Tikhonov N.A., 1994, A\&Ap 286, 718

Kraan-Korteweg R.C., 1986, A\&AS 66, 255

Landolt A.U., 1992, AJ 104, 340

McCall M.L., Rybski P.M., Shields G.A., 1985, ApJS 57, 1

McCall M.L., 1995, (private communication)

Natali G., Pedechini F., Reghini M., 1992, A\&A 256, 79

Paturel G., Bottinelli L., Fouque P., Gouguenheim L., 1992, Catalogue of Principal Galaxies: PGC-ROM, Observatoire de Lyon

Piotto G., Capaccioli M., Bresolin F., 1992, Mem. S. A. It. 63, 465

Rozanski R.,Rowan-Robinson M., 1994, MNRAS 271, 530

Thuan T.X., Seitzer P.O., 1979, ApJ 231, 680

Tikhonov N.A., Drozdovsky I.O., 1996, Astrophys. Lett. Comm. (submitted)

Treffers R.R., Richmond M.W., 1989, PASP 101, 725

Tully R.B., 1987, ApJ 321, 280

Tully R.B., 1988, Nearby Galaxies Catalogue, Cambridge. Cambridge Univ. Press

Sandage A.,Bedke J., 1988, Atlas of Galaxies, Useful for Measuring the Cosmological Distance Scale, NASA, Washington

Sandage A.R., Tammann G.A., 1974a, ApJ 190, 525

Sandage A.R., Tammann G.A., 1974b, ApJ 191, 603 
Sandage A.R., Tammann G.A., 1974c, ApJ 194, 223

Sandage A.R., Tammann G.A., 1974d, ApJ 194, 559

van den Bergh S., 1966, AJ 71, 922

Vennik J., 1984, Tartu Astron. Obs. Publ. 73, 1

von Hippel T., Bothun G., 1990, AJ 100, 403

Wevers B.M., 1984, Ph. D. Thesis, Groningen 